\title{
Translating Museum Visual Contents into Descriptions for Blind Users: a Multidisciplinary approach
}

\author{
Barbara Leporini $^{1}$ and Ivan Norscia ${ }^{2}$ \\ (1) ISTI - CNR, Pisa - Italy \\ barbara.leporini@isti.cnr.it \\ ${ }^{(2)}$ Museo di Storia Naturale e del Territorio - Università di Pisa \\ norscia@lunet.it
}

\begin{abstract}
Accessibility and usability guidelines are available to design web sites accessible to blind users. However, the actual usability of accessible web pages varies depending on the type of information the user is dealing with. Museum web sites, including specimens and hall descriptions, need specific requirements to allow vision-impaired users, who navigate using a screenreader, to access pieces of information that are mainly based on visual perception. Here we address a methodology to be applied for the proper creation and elaboration of alternative image descriptions in museum web pages. Such methodology has been applied to a gallery of the Museum of Natural History and Territory (University of Pisa). Such indications allow the user: (1) to address indexed contents and to link to information in more details, (2) to calibrate image descriptions (with a command providing alternative explanations for specimens), and (3) to access extra information for the blind (via hidden labels). A multidisciplinary approach is necessary to obtain effective and comprehensive descriptions. In this perspective, a cooperative environment is eventually proposed for team work facilitation.
\end{abstract}

Keywords: virtual visit, guidelines, museum, cooperation, accessibility, usability

\section{Introduction}

Most museums make part of their content available in their websites, for selfadvertisement and to light up the interest of potential visitors. An online preliminary visit can help planning the real visit and/or provide in-depth and complementary information to be used for educational purposes.

While the preliminary visit can improve the quality of the visit for "regular" users, it can represent the key of a successful visit for special users, such as blind people. In 
fact, through an online pre-visit, blind users have the possibility a) to prepare a "mental plan" of the spatial display of museum specimens, exhibitions, and halls; and b) to start dealing the museum contents, in order to avoid information overload during the real visit. The informational gap experienced by blind users (visual display and content location) cannot be properly filled by museum operators, who are constrained by the short time of an average visit. Since time constrains clash with multifaceted information, the visit may eventually result in a simple specimen list or in a sloppy presentation of the overall content.

In order to be really fruitful and effective for disabled users, the virtual visit must be not only well organized and appealing but also accessible. Although a plethora of guidelines, criteria, and suggestions have been proposed to gain or increase web accessibility and usability [3, 6, 7, 8, and 12], a recent international survey of 125 museum Web sites showed that only a minority of them fulfilled the basic accessibility requirements [5]. Consistently, user trials revealed that a non negligible part of visually and cognitively impaired users was not able to recuperate pieces of information or accomplish simple tasks on most of the Web sites included in the survey [5].

Museum web sites need additional accessibility requirements to allow visionimpaired users, who navigate using a screen-reader, to access contents that are largely based on visual perception. A crucial aspect for the suitability of museum web sites is image description, since pictures, figures, and illustrations are an important means for understanding properties and value of scientific specimens, pieces of art, or collections. Of course, comprehensive and effective descriptions (including visual cues, notions, and cultural meaning) can be provided by a combination of experts in different fields (e.g. biology, history, web designer, photography, accessibility).

In this article we address the issue of image description in museum web sites by presenting the case of the Museum of Natural History and Territory of the University of Pisa. Here we propose a multidisciplinary approach, in order to overcome the problem of content accessibility prevented by the imprecise, or even missing, translation of the image content into screen reader language (descriptive text). The article is organized as follows: introduction (present section), theoretical problem (section 2), methodology (section 3), case study (section 4), possible solution via cooperative approach (section5).

\section{The problem}

Accessibility and usability guidelines should allow the creation of web sites suitable for disabled users. However, most guidelines have been created considering accessibility as invariant with respect to content type. Instead, actual usability and accessibility of web pages varies depending on the type of information the user is 
dealing with. As a consequence, the guidelines are often loose and their successful application is too much dependent on personal interpretation.

For example, the checkpoint 13.1 "Clearly identify the target of each link" reported in the WCAG 1.0 guidelines for accessibility [8], states that "Link text should indicate the nature of the link target", providing indications like "more information about sea lions" or "text-only version of this page" rather than "click here" or "more information". Nevertheless, a study conducted on 100 web sites reported that link description is one of the accessibility problems still encountered during web navigation [4].

A further controversial issue is represented by the application of alternative descriptions to non-text elements, as indicated by the checkpoint 1.1 "Provide a text equivalent for every non-text element (e.g., via "alt", "longdesc", or in element content)" [8]. Based on this checkpoint, web designers should apply different levels of description, short, more extensive, or alternative. However, the application is controversial since the type of description cannot be univocally predicted basing on the typology of non-text elements, as in the case of images.

For example, no long description needs to be associated to images if they refer to products sold via e-commerce: in this case, the blind customers are more interested in identifying the item they are willing to buy more than in getting an in-depth description of the visual features of the product. In this case, short and clear labels are welcome. On the contrary, the visual features of a scientific specimen or a piece of art are necessary to understand their intrinsic value and properties, thus a detailed and more comprehensive image description is in order here.

Although possible solutions have been proposed to help web designers to create alternative descriptions [1], assigning a proper description remains a difficult task, since web designers must determine, as a first step, the underlying role of an image. In a Museum web site at least three situations are likely to occur. An image can represent a link (e.g. to the home page) and the relative alternative description falls more into checkpoint 13.1 than into checkpoint 1.1 [8]. In this case it is more appropriate to label the image function (e.g. "home page") than providing a description of the image itself, which can reported (once for all) in the home page. An image can also be purely ornamental (e.g. pictures of Museum building): in this case a short description is probably enough. Finally, pictures or illustrations can be related to museum specimens and, consequently, the description must be extended since such elements are necessary to understand web page content.

Alternative descriptions can be classically assigned via “alt” attribute (short text), "longdesc" attribute (longer descriptions), or an external link addressing an entire explanatory page. Here we provide a new option to insert longer descriptions without the use of an external link or the "longdesc" attribute. Since description typology varies according to the type of specimen or piece of art described, a multidisciplinary approach must be considered in order to make such new option effective. 


\section{The methodology}

In order to make the online visit both accessible and usable it is important that image location be well organized. Often many images and videos, with relative information, are clumped in a single page because they are related to a specific exhibition or hall. Even if such organization can make sense for "regular" users, who are able to detect both the image set and the whole visual layout all at once, vision impaired users can be disoriented because they process the contents in a sequential way, via screen reader [12]. Scrolling down all the images is not only time consuming but also confusing and unpractical during information search. Instead, a list of short descriptions of visual items could allow vision impaired users to pick up specific items only on request.

In this view, web pages, each one referring to a hall/gallery, can be organized according to two levels: 1) a summary listing the items exhibited and their location in the hall (index page) and 2) a descriptive page for each items (item detail page), which can be entered on request by linking on each piece listed in the index page.

- Index page - here the following information is reported:

o "Welcome description" - a summary of the main content of the hall/exhibition.

o "Memo description" - a reminder for blind users on how to use the page.

o "Spatial description" - a map to help orientation in the hall.

o "Inventory description" - List of the items with a link to a new window (item detail page, see below).

- Item detail page - Here all information about a piece of art or specimen is reported, well separated from any other confounding element. Vision impaired users have the possibility to concentrate on a single item and, if not totally blind, they can use specific software to magnify and navigate the page. The page is organized as following:

o Magnified image - a picture with a short description suitable for exploration by low vision users.

o Notional Caption - In-depth description for all users. This part provides information on origin, nature, meaning, and cultural relevance of the item.

o Visual caption: description of visual cues important for a complete understanding of the presented items. Such information can be read via screen reader only. Colours, shapes, spatial dislocation (if the item is composed by different elements) and other features appearing in the image (e.g. if an animal is hairy or not) need to be included in this description. It should be noticed that this part does not carry any background information on the item itself. As a matter of fact it has nothing to do with the 
notional caption (necessary to all users). The visual caption only provides visual cues that a blind user would not get otherwise.

The possibility of inserting the different types of descriptions must be considered by web designers since the beginning, in order to integrate all the pieces of information in a clear and well structured way. However, notional and visual descriptions must be scrupulously prepared by experts in the field, and not by web designers, because details are important for the analysis of scientific specimens (e.g. animal or plant reconstructions) or pieces of art (e.g. a marble statue). Moreover, since a museum can include different disciplinary fields (e.g. paleontology, zoology, botany), several experts can be required to provide appropriate descriptions and information to all the items shown in the museum web site.

In this perspective, the team work for web site realization is needed and it should involve the following people:

I. Web site designer, for the creation and the technical development of the web site.

II. One or more experts in the field, for providing item descriptions.

III. A coordinator, who should gather and integrate the information prepared by the experts.

IV. A sample of final users with special needs, to test the web site via assitive technology tools (e.g. screen reader and magnifier).

\section{The case study}

When we focus on the web page describing a specific exhibition/hall, the questions to address are:

- how to structure the index page containing the information on the exhibition/hall and the list of specimens that can be "explored" during the visit;

- how to organize the item detail page assigned to each specimen image;

- how to create and insert the additional description to be used by vision impaired users.

In order address such questions, we referred to the web site of the Museo di Storia Naturale e del Territorio, Università di Pisa and, specifically, to the page describing the gallery of human evolution and early hominids (home $>$ esposizioni $>$ zoologia $>$ ominidi). Such gallery includes only four different dioramas (partially three dimensional models), thus representing an ideal model to implement, for the first time, a new methodology.

Following the methodology proposed in section 3, we structured the index page as follows: 
- A "welcome description", a short opening part reporting main subject (a sort of an expanded title); in our case: "The gallery of human history presents human evolution from early hominids to $\underline{\text { Homo }}$ sapiens".

- A "memo description", for users navigating through screen reader; this part reminds the users that they can click on each item link (reported below in the page) to open a new window containing an in-depth explanation and a specific hidden description for the blind.

- A "spatial description" reporting hall shape (square), size (20 m x 20m) and the location of the four dioramas (in this case one per each side); this part is important for orientation and can be analysed before the visit. In bigger exhibitions this part can take the form of a map, with its own detail page associated.

- An "inventory description", reporting a list of diorama titles: 1) the study of an anthropologist of XIX century, 2) Laetoli footprints and Australopithecus afarensis in the African savannah 3) Homo neanderthalensis in an everyday life scene, and 4) drawings by early Homo sapiens from the Chauvet cage. Each title is associated with a small image, which can be clicked on to get into a new window page dedicated to the diorama (see Figure 1 below).

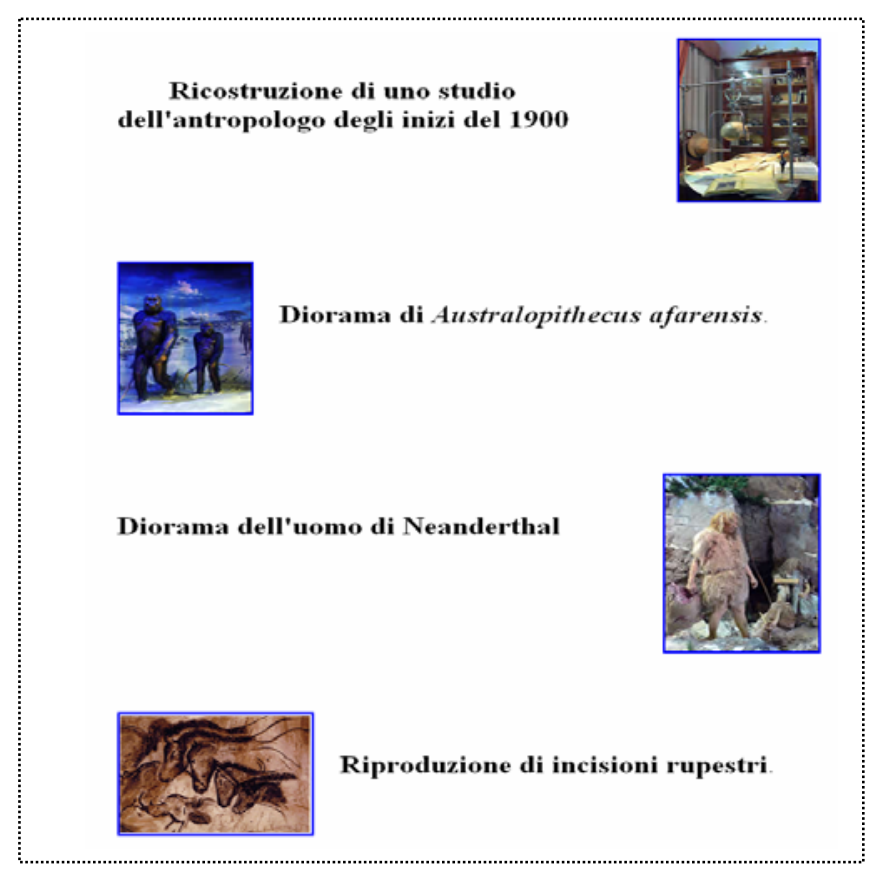

Figure 1 - List of items in the index page.

Subsequently, we organized the item detail page by including 1 ) the diorama picture (magnified image), 2) an explanation for all users (notional caption), and 3) a hidden description for vision impaired users (visual caption). We inserted the 
alternative description for blind users via hidden labels. Normally, when the description is not short the attribute "longdesc" or a descriptive link ("[D]" type) are used. However, when the description is rather complex and multifaceted, the navigation procedure can become confusing and misleading. Consequently, we decided to insert an alternative description that can be read only by the screen reader (thus hidden to "regular" users), so that the vision impaired users can find all the information in the same page. Figure 2 (below) shows an example of item detail page for the exhibition considered here.

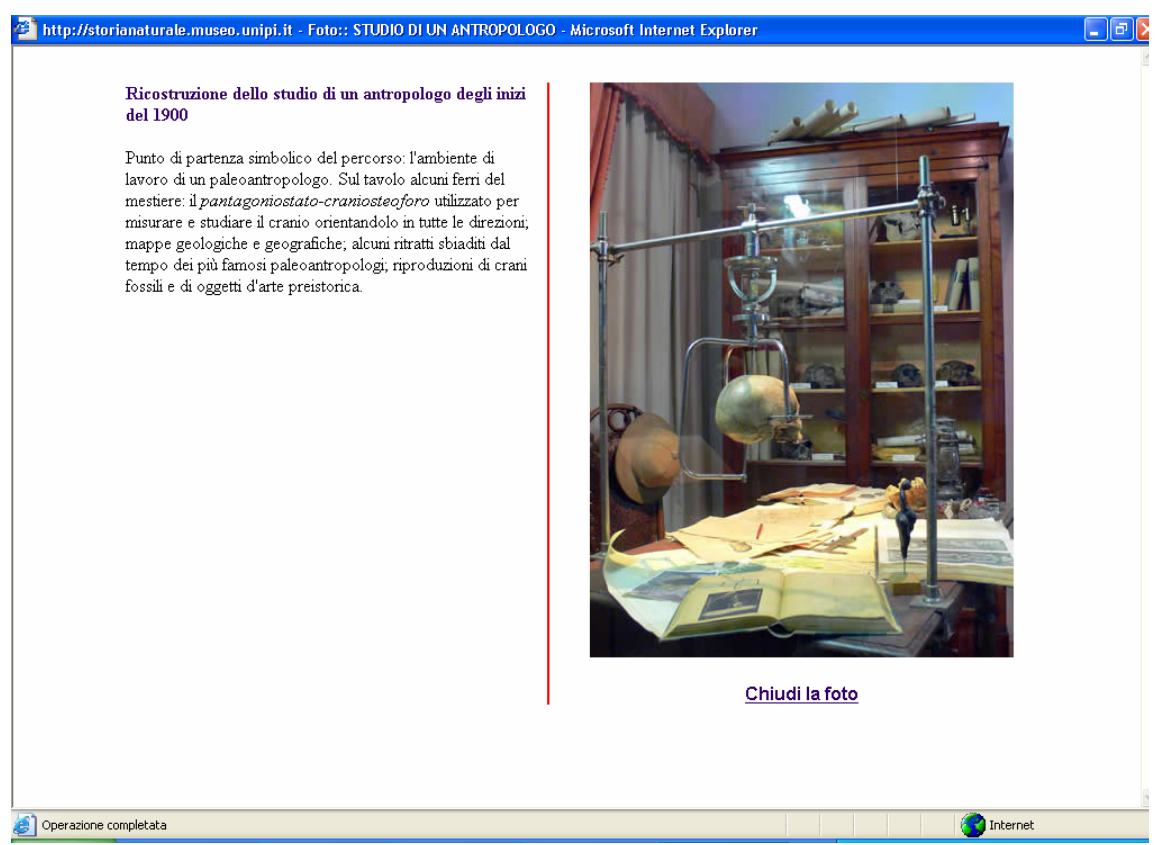

Figure 2 - An example of a item detail page

The detail page associated to diorama 1 (Figure 2) can be opened by clicking on the correspondent diorama small image contained in the index page. Such small image is associated to the link text "study of an anthropologist: picture - link to a new window". The part "link to a new window" is inserted only if Javascript is active, otherwise the detail page will open in the main browser window.

Figure 3 (below) shows the html code used for the visual caption (left) and the information sequence read by the screen reader (right). It should be noticed that the screen reader detects, as first, the presence of the image with the alternative short label, then the hidden description and, eventually, the general explanation appearing on the left side of the window. Such sequence, different from the order that can be detected by the visual layout, is caused by the HTML code flow and by the CSS 
properties used for page editing. We decided to give priority to the hidden description because the picture itself could contain elements that are important to understand the explanation. In this specific case, it is crucial to know that a clamping metal device is located on the table, in order to understand the general explanation.

\begin{tabular}{|c|c|}
\hline $\begin{array}{l}\text { //CSS fragment } \\
\text {.hidden-label \{position:absolute; left:- } \\
\text { 1000em; z-index:-1;\} } \\
\text {.. } \\
\text { //HTML fragment }<\text { div id="rightfoto" }> \\
<\text { img src="../images/photo.jpg" } \\
\text { alt="the study of an anthropologist" } /> \\
</ \text { div }><\text { !-- fine foto--> } \\
<\text { div id="leftfoto"> } \\
<\text { p class="hidden-label" }>\text { Picture } \\
\text { alternative description: notes, papers, } \\
\text { and research books lie chaotically upon } \\
\text { a wood desk...</p }> \\
<\text { p }>\text { This is starting point of our } \\
\text { "journey" through } \\
\text { evolution... }</ \text { p }> \\
</ \text { div }>\quad\end{array}$ & $\begin{array}{l}\text { Photo:: The study of an anthropologist } \\
\text { Graphic: the study of an } \\
\text { anthropologist } \\
\text { Reconstruction of the typical study of } \\
\text { an anthropologist of the XIX century. } \\
\text { Picture alternative description: } \\
\text { notes, papers, and research books lie } \\
\text { chaotically upon a wood desk, in } \\
\text { front of you... A skull, which seems } \\
\text { suspended in the air, is sort of } \\
\text { clamped by two arms of a metal } \\
\text { device... On the backside, various } \\
\text { reproductions of hominid skulls are } \\
\text { located on shelves... } \\
\text { End of alternative description } \\
\text { This is the starting point or our } \\
\text { "journey" through human evolution: } \\
\text { the working place of a paleo- } \\
\text { anthropologist. the particular device } \\
\text { upon the table is used in physical } \\
\text { anthropology for craniometric } \\
\text { measurements... } \\
\text { Link shut the window }\end{array}$ \\
\hline
\end{tabular}

Figure 3 - HTML and CSS code (on the left, A); Page portion read by the screen reader (on the right, $B$ ). 


\section{Preliminary user-based evaluation}

We conducted a preliminary test in order to evaluate if the application of our criteria was successful in allowing blind users to collect information that they could not obtain otherwise. The test involved 16 blind users with similar computer skills (they were all participating to the same computer training class, at the intermediate level). They were asked to perform a virtual visit to the gallery of human evolution and early hominids: half of them were asked to navigate an accessible site without our additional criteria while the other half used the site including our criteria. After a fixed navigation time (15 minutes) they were asked to answer to five questions included in a multiple choice questionnaire. The questions were relative to basic information that could be obtained by both pictures (with the short alternative description always included) and text. A score of 5 points was assigned for each exact answer. On average, the users navigating the implemented site could obtain a higher score (mean \pm standard error: 20.63 \pm 5.63) than the users using the site without additional criteria (mean \pm standard error: 6.88 \pm 4.58 ). Even if quantitative and more in-depth analyses are in progress, this preliminary result suggest that our additional criteria are effective in expanding the background information that a blind user can obtain during the virtual visit of a museum gallery.

\section{Multidisciplinary approach in a cooperative environment}

An integrated cooperative environment on the web is important to enhance and improve the team work required to plan, organize, develop, and successfully implement museum web pages (a working page is shown in Figure 4). 


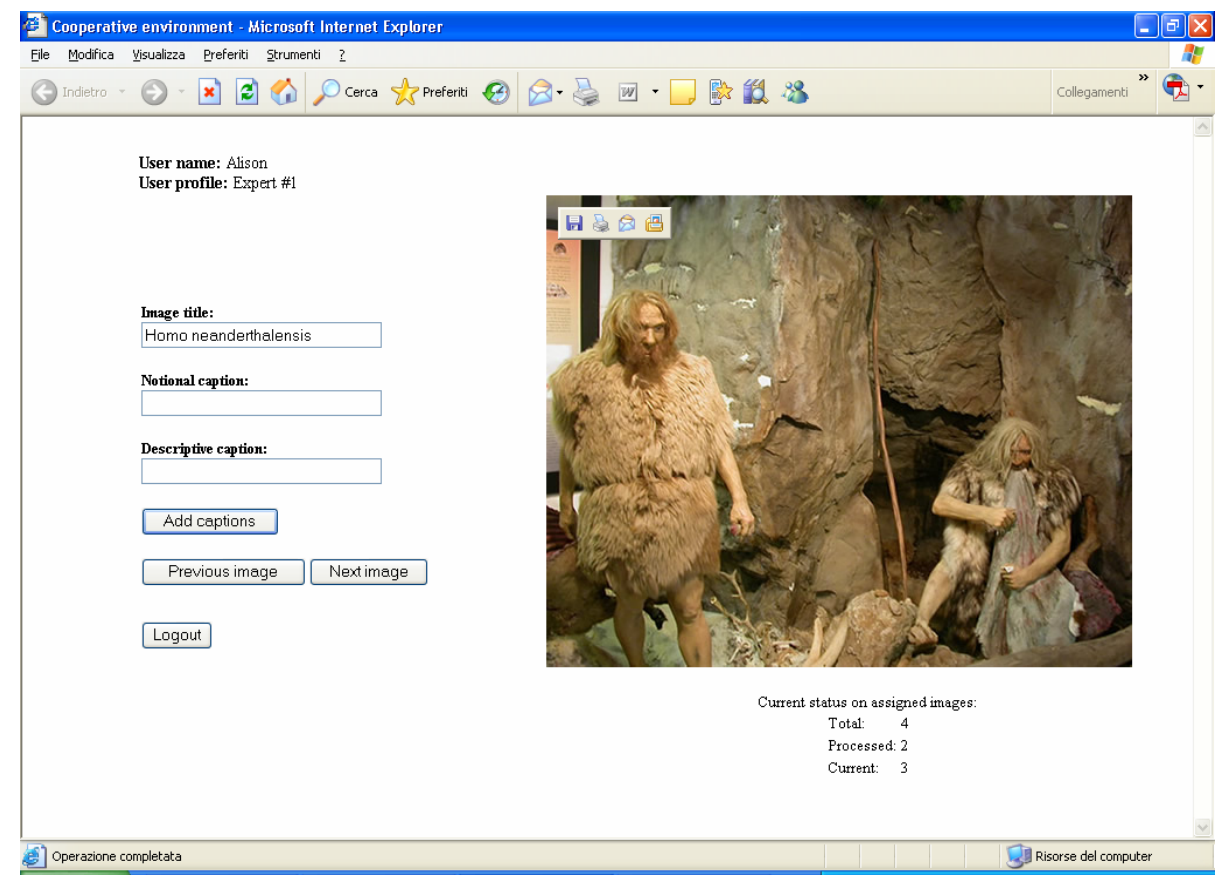

Figure 4 - A working page in a cooperative environment

Such environment needs to have the following features:

- Web-based system. The environment must be created via Web technology and accessible from everywhere. In this way, web site creation is relieved by the necessity of having all team members in geographical proximity. An immediate consequence would be a wider array of options for team members selection. In our case study, the experts in different fields could cooperate from there "home base": Department of Anthropology, C.N.R., Museum of Calci, etc.

- User profile differentiation. The environment needs to include different access profiles, following an identification/authentication process, in order to separate system subsets, differing according to the role of each team member. In this case study, a web designer, a Museum expert, a photographer, an anthropologist, and a C.N.R. researcher entered the system via different access profiles.

- Content-based database. The environment is based on a database gathering all the pieces of information provided by the team members. In our case, for example, the Museum expert provided information on the exhibit (spatial organization and dioramas), the anthropologist entered biological information on the early hominids recreated in the hall, and the 
C.N.R. researcher managed ensure that accessibility criteria were followed.

- Collaborative \& cooperative interaction. The environment should allow team members to communicate and exchange information with each other, in order to allow proper integration, combination, and connection of all contents.

Collaboration and cooperation are crucial in order for the content i) to remain consistent throughout the site, ii) to be uniformly organized, and iii) to maintain overall homogeneity.

\section{Conclusions}

In the present work, we addressed the important issue of accessibility and usability of Museum web sites that should allow online visits suitable for vision impaired users navigating through screen reader. In particular, we provided a methodology to create a system of additional and alternative descriptions able to present, appropriately, scientific specimens and art pieces. Such methodology, applied on the web pages of a museum, has been studied to provide complements of information without complicating the web page framework, thus avoiding confusion and disorientation.

We also stressed out the necessity of a team of experts, whose expertise in different topics must be combined to provide effective and useful image descriptions to web designers, who will integrate in the web site. The problem of scientific image description, requiring precision, competence, and expertise, goes beyond web sites and also involve books and all the scientific material addressed to blind users (tools, specimens, etc.). The cooperative environment proposed to create Museum web sites could be easily recycled for any type of elaboration involving scientific subjects.

\section{Acknowledgements}

The authors wish to thank Walter Landini and Elisabetta Palagi (director and curator of the vertebrate section, respectively) of the Museum of Natural History and Territory (University of Pisa). 


\section{References}

1. Ahn, L., Ginosar, S., Kedia, M. Liu, R., Blum, M. (2006). Improving Accessibility of the Web with a Computer Game. In the Proceedings of the SIGCHI conference on Human Factors in computing systems. Montréal Québec, Canada April 22 - 27, 2006. pp. 79 - 82

2. Leporini B., Paternò F. (2004). Increasing usability when interacting through screen readers. In: International Journal Universal Access in the Information Society, Vol. 3 n. 1 (2004), pp. 57-70. Springer Verlag, 2004.

3. Leporini B., Paternò F. (2008). Applying Web Usability Criteria for Vision-Impaired Users: Does It Really Improve Task Performance? In: International Journal of Human-Computer Interaction, vol. 24 (1) pp. 17 47. Taylor \& Francis, 2008.

4. Petrie, H., Hamilton, F., King, N. (2004). Tension, what tension?: Website accessibility and visual design. In the Proceedings of the 2004 international cross-disciplinary workshop on Web accessibility (W4A), ACM International Conference Proceeding Series; Vol. 63, pp. $13-18$

5. Petrie, H., King, N. and M. Weisen, The accessibility of museum Web sites: results from an English investigation and international comparisons. In J. Trant and D. Bearman (eds.). Museums and the Web 2005: Proceedings, Toronto: Archives \& Museum Informatics, published March 31, 2005 at http://www.archimuse.com/mw2005/papers/petrie/petrie.htm

6. Stephanidis, C., Akoumianakis, D., Sfyrakis, M., \& Paramythis, A., 1998. Universal accessibility in HCI: Process-oriented design guidelines and tool requirements. In C. Stephanidis \& A. Waern (Eds.), Proceedings of the 4th ERCIM Workshop on "User Interfaces for All", Stockholm, Sweden, 19-21 October .

7. Theofanos, M.F., Redish, J., 2003. Bridging the gap: between accessibility and usability. ACM Interactions magazine, New York: ACM Press, Nov.-Dec. 2003 issue, pp.36-51

8. Web Content Accessibility Guidelines 1.0 URL: http://www.w3.org/TR/WAI-WEBCONTENT

9. Web Content Accessibility Guidelines 2.0 Draft December 2007 - January 2008 URL http://www.w3.org/WAI/GL/WCAG20/WD-WCAG2020071218/ 